Canadian

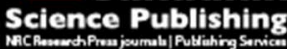

Applied Physiology, Nutrition, and Metabolism Physiologie appliquée, nutrition et métabolisme

\title{
Association between exercise-induced change in body composition and change in cardio-metabolic risk factors in post-menopausal South Asian women
}

\begin{tabular}{|r|l|}
\hline Journal: & Applied Physiology, Nutrition, and Metabolism \\
\hline Manuscript ID & apnm-2016-0082.R2 \\
\hline Manuscript Type: & Article \\
\hline Date Submitted by the Author: & 20 -Apr-2016 \\
\hline Complete List of Authors: & $\begin{array}{l}\text { Lesser, Iris; Simon Fraser University, Biomedical Physiology and } \\
\text { Kinesiology } \\
\text { Guenette, Jordan; University of British Columbia, Physical Therapy } \\
\text { Hoogbruin, Amandah; Kwantlen University } \\
\text { Mackey, Dawn; Simon Fraser University } \\
\text { Singer, Joel; University of British Columbia, School of Population Health } \\
\text { Gasevic, Danijela; Simon Fraser University, Biomedical Physiology and } \\
\text { Kinesiology } \\
\text { Lear, Scott; Simon Fraser University, }\end{array}$ \\
\hline Keyword: & $\begin{array}{l}\text { visceral fat < waist circumference, ethnicity, exercise < exercise, } \\
\text { cardiovascular risk factors }\end{array}$ \\
\hline
\end{tabular}

\section{SCHOLARONE}

Manuscripts 
Association between exercise-induced change in body composition and change in cardiometabolic risk factors in post-menopausal South Asian women

Lesser Iris $\mathrm{A}^{1}$, Guenette Jordan $\mathrm{A}^{2}$, Hoogbruin Amandah ${ }^{3}$, Mackey Dawn $\mathrm{C}^{1,4}$, Singer Joel ${ }^{5}$, Gasevic Danijela ${ }^{6}$, Lear Scott $\mathrm{A}^{1,7,8}$

1. Department of Biomedical Physiology and Kinesiology, Simon Fraser University, Vancouver, BC, Canada V6B 5K3

2. Department of Physical Therapy and Centre for Heart Lung Innovation, University of British Columbia and St Paul's Hospital, Vancouver, BC, Canada V6Z 1Y6

3. Faculty of Health, Kwantlen Polytechnic University, Surrey, BC, Canada, V3W 2M8

4. Centre for Hip Health and Mobility, University of British Columbia, Vancouver, BC, Canada V5Z 1M9

5. School of Population and Public Health, University of British Columbia and St. Paul's Hospital, Vancouver, BC, Canada V6Z 1 Y6

6. Centre for Population Health Sciences, University of Edinburgh, Edinburgh, United Kingdom EH8 9AD

7. Faculty of Health Sciences, Simon Fraser University, Burnaby, BC, Canada V5A 1S6

8. Division of Cardiology, Providence Health Care, Vancouver, BC, Canada V6Z 1Y6

\section{Corresponding Author}

Scott A. Lear

Healthy Heart Program, St. Paul's Hospital

180 - 1081 Burrard Street

Vancouver, BC, V6Z 1Y6

slear@providencehealth.bc.ca

604-682-2344 ext 62778 


\section{Abstract}

The South Asian (SA) population suffers from a high prevalence of type 2 Diabetes and cardiovascular disease (CVD). A unique obesity phenotype of elevated visceral adipose tissue (VAT) is associated with CVD risk among SA. Exercise-induced reduction in VAT and body fat is an effective mechanism to improve cardio-metabolic risk factors but this has not been shown in SA. Whether exercise-induced changes in measurements such as waist circumference (WC) are independently related to changes in cardio-metabolic risk factors in SA is unknown. Multi-slice computed tomography scanning was used to assess VAT, cardiometabolic risk factors through a fasting blood sample and body fat using dual energy x-ray absorptiometry. Forty- nine post-menopausal South Asian women who participated in two 12-week aerobic exercise programs were included. Bivariate correlations were used to assess associations between change in cardio-metabolic risk factors and change in body composition. Regression analyses were conducted with change in glucose, insulin and homeostatic model assessment of insulin resistance (HOMA-IR) as dependent variables and change in body composition as independent variables of interest. There were significant associations between changes in fasting insulin, glucose and HOMA-IR with change in VAT. The association between change in VAT and these cardio-metabolic risk factors was independent of change in other body composition variables of interest. South Asian women should be encouraged to engage in aerobic activity to reduce their risk of type 2 diabetes and CVD, and physicians should be aware of improvements in glucose regulation with exercise training not observed through reductions in $\mathrm{WC}$.

Keywords

Ethnicity; Exercise; Visceral Fat; Waist Circumference; Cardio-metabolic risk factors; South Asian 
Introduction

Globally South Asians make up one quarter of the world's population and are a sizeable ethnic group in many Western countries. South Asian ethnicity is associated with a greater risk of type 2 diabetes (T2D) (Gholap et al., 2011) and cardiovascular disease (CVD) (Garduno-Diaz, Khokhar 2011), which may be due to the unique and deleterious South Asian obesity phenotype of greater body fat, greater visceral adipose tissue (VAT), and lower lean body mass compared to Europeans (Lear et al. 2007). The South Asian obesity phenotype explains a large amount of the ethnic variation in insulin sensitivity, suggesting a role of excess body fat in elevated T2D and CVD risk (Lear et al., 2009). Further, elevated cardio-metabolic risk factors in South Asians, such as (cholesterol and glucose), are largely explained by greater amounts of VAT than Europeans (Lear et al. 2012). Of particular risk among the South Asian population are women after menopause, as studies in postmenopausal women of other ethnicities have demonstrated that menopause is associated with increased risk for T2D and CVD (Rosano et al., 2007). This also appears coincident with changes in body composition that include enlargement of the VAT depot (Janssen et al., 2015).

Both reductions in VAT and total body fat have been suggested as primary targets (Janiszewski and Ross, 2009) for reducing the prevalence of T2D and CVD in the South Asian ethnic group and specifically post-menopausal women. While removal of VAT through surgical means has proven unsuccessful at altering cardio-metabolic risk factors (Fabbrini et al. 2010), aerobic exercise is effective at reducing VAT and total body fat while improving cardio-metabolic risk in European populations (Arsenault et al. 2009, Bouchonville et al. 2014) due to the preferential reduction of VAT through alpha adrenergic activation which 
occurs with aerobic exercise (Arner et al. 1990) due to greater sensitivity to lipolytic catecholamines (Fried et al., 1993).

A reduction in VAT may explain exercise-induced improvement in cardio-metabolic risk factors (Borel et al., 2012) but direct measurements of abdominal adipose tissue and total body fat are difficult and expensive to obtain and may expose a patient to radiation; therefore, simple anthropometric measures to overcome these feasibility issues are needed. Waist circumference (WC) has been identified as a simple anthropometric clinical marker of VAT, and a larger WC is associated with elevated CVD risk (Despres 2014). It has been suggested that associations between VAT and WC remain after weight loss; (Pare et al., 2001) however, whether WC is effective for determining VAT-derived improvements in CVD risk is unclear given that change in WC could represent a combination of a change in subcutaneous abdominal adipose tissue (SAAT) in addition to a change in VAT (Lemieux et al. 2000). In addition, it is unknown whether exercise-induced change in body composition alters CVD risk in post-menopausal South Asian women as previously seen in European populations (O’Leary et al., 2006; Nicklas et al., 2009).

We have previously found in within-group pre-post comparisons, that there was a significant reduction in VAT $(\mathrm{p}=0.040)$ and WC $(\mathrm{p}=0.037)$ after a 12-week standard exercise program, as well as in SAAT $(\mathrm{p}<0.001)$, TAAT $(\mathrm{P}<0.001)$, BMI $(\mathrm{p}=0.027)$, WC $(\mathrm{p}<0.001)$, body fat $(\mathrm{p}=0.036)$ and glucose $(\mathrm{p}=0.039)$ after a 12-week Bhangra dance program (Lesser et al., 2016). Therefore, our primary objective in this study was to assess the associations between changes in VAT, SAAT, WC, percent body fat and BMI with changes in cardiometabolic risk factors following a 12-week supervised aerobic exercise intervention in inactive, post-menopausal South Asian women as a follow-up to the above mentioned randomized controlled trial. Our secondary objective was to assess whether the associations between changes in VAT and total body fat and change in cardio-metabolic risk 
were independent of changes in WC and BMI, respectively. Our tertiary objective was to explore whether the measurement of VAT and total body fat would explain variability in changes in cardio-metabolic risk (CMR) over and above the changes in WC and BMI, which are tools commonly used in clinical practice.

\section{MATERIALS AND METHODS}

\section{Study Population}

This study was a secondary analysis of data from a registered randomized controlled trial (ClinicalTrials.gov \#NCT01766453). Participants were women recruited from the South Asian community in the Greater Vancouver area through local media: television, radio, newspapers, local temples, community events, and word of mouth. Women were eligible to participate in the study if they were post-menopausal (one year since their last menstrual cycle), had not engaged in regular physical activity in the previous 6 months, and had a waist circumference of $80 \mathrm{~cm}$ or greater (cut off for abdominal obesity for South Asian women) (Alberti et al. 2005). Individuals were not eligible to participate if they reported having been diagnosed with CVD or T2D. All participants provided written informed consent, and this study was approved by the Simon Fraser University and Providence Health Care Research Ethics Boards.

\section{CT Scanning for Abdominal Adiposity}

Multi-slice computed tomography scanning was used to assess VAT volume. After an initial image acquired at the L4-L5 interverterbral disk, three $10 \mathrm{~mm}$ slices were taken every $5 \mathrm{~cm}$ above this landmark and two below this landmark. Scan parameters were set at $120 \mathrm{kVp}$, $300 \mathrm{~mA}$ for one second, $10 \mathrm{~mm}$ thickness, 512 by 512 matrix using a $48 \mathrm{~cm}$ field of view. All six CT slices were obtained at the same time. Using the standard attenuation range of -190 to -30 Hounsfield units for adipose tissue, the cross-sectional areas were determined using 
imaging software (SliceOmatic v4.2 Tomovision, Montreal, Quebec). VAT was calculated as all pixels in this attenuation range within the inner abdominal wall. Volumes were calculated as the average of the two closest scans multiplied by the distance between the scans. All areas were then added together for volume. We additionally assessed total abdominal adipose tissue (TAAT) calculated as all pixels within this attenuation range in the abdominal image and subcutaneous abdominal adipose tissue (SAAT) calculated as the difference between TAAT and VAT. Scan analyses were completed by the same individual, with a coefficient of variation for the analysis of $1.21 \%$ at the L4-L5 scan. All scans were void of participant identity prior to assessment to avoid any interpretation bias.

\section{Body Composition Analysis}

Mass in kilograms and height in metres were assessed with participants in light clothing (or hospital gowns), footwear removed and pockets emptied. Body mass index was calculated as mass in kilograms over height in metres squared. Waist circumference was recorded in centimetres as the average of two measures taken against the skin at the point of maximal narrowing from the anterior view following a normal expiration. Dual energy x ray absorptiometry (DXA) (Hologic Discovery QDR 4500w, Bedford, Massachusetts) was used to determine lean body mass $(\mathrm{kg})$, total body fat mass $(\mathrm{kg})$, and percent body fat (calculated by dividing total body fat by total body mass).

\section{Cardio-metabolic Risk Factor Assessment}

Cardio-metabolic risk factors were measured after a 12-hour overnight fast and 48-hour abstinence from alcohol. Blood samples were collected by venipuncture and immediately processed and assessed. Serum and plasma assays (total cholesterol, high density lipoprotein cholesterol, non high density lipoprotein cholesterol, triglycerides, glucose, insulin, apolipoprotein A, apolipoprotein B, aluminotransferase, C-reactive protein) were 
conducted by standard procedures in the St. Paul's Hospital Laboratory, Vancouver, Canada. Method accuracy, inter- and intra-assay precision met the stringent criteria required by the Canadian External Quality Assessment Laboratory. Low density lipoprotein cholesterol was assessed using the Friedewald equation (Friedewald, Levy \& Fredrickson 1972). Homeostatic Model Assessment of Insulin Resistance (HOMA-IR) was calculated as fasting glucose multiplied by fasting insulin divided by 22.5 (Matthews et al., 1985).

\section{Exercise Testing}

Participants completed a treadmill test using the Bruce Protocol (Bruce, Kusumi \& Hosmer 1973). The test began at $1.7 \mathrm{mph}$ and a $10 \%$ grade with the speed and grade increasing progressively until the participant reached volitional exhaustion. Prior to testing each participant, a gas calibration and volume calibration was completed using standard procedures. Heart rate was assessed by ECG throughout the test for safety purposes. Metabolic data were collected simultaneously during the treadmill test on a breath-bybreath basis using a calibrated metabolic system (VMax SensorMedics, San Diego, California). Peak oxygen uptake $\left(\mathrm{VO}_{2}\right.$ peak)was acquired as the highest 20 -second average at the end of the test. Participants were encouraged verbally throughout the test in an attempt to achieve a maximal effort. Time to exhaustion was measured by time spent on the treadmill protocol prior to volitional exhaustion.

\section{Exercise Intervention}

Participants included in this study participated in either a progressive standard aerobic exercise program or a Bhangra dance program, 3 times per week for 12 weeks and these two groups were combined for the analyses as both exercise groups showed the same direction of change in variables of interest. Participants were allocated to either; control, standard exercise or Bhangra dance using variable block sizes of 2,4 and 6 . The random 
allocation was computer-generated by a statistician unassociated with the trial. The exercise group was not compared to the original control group (a third group who were instructed to maintain current lifestyle habits) as the intention was to denote associations with change in body composition and change in cardio-metabolic risk factors post exercise intervention. The standard aerobic exercise program consisted of a 10-minute group warm up, 40 minutes of aerobic conditioning and a 10-minute group cool down. The prescribed exercise intensity was individualized based on maximal heart rate achieved during the exercise test. Heart rate (HR) was prescribed at 55\% of HRmax and increased $10 \%$ every 3 weeks with the last 3 weeks of the program prescribed at $85 \%$ of HRmax. HR was assessed by the personal trainer every 10 minutes of the conditioning period and the exercise intensity was adjusted if necessary. The Bhangra dance program consisted of a 10-minute group warm up, 40 minutes of Bhangra dance and a 10-minute group cool down. Bhangra dance is a form of folk dance which originates from the Punjab area of India and consists of high intensity jumps, kicks and upper body movement to Bhangra music. The intensity of the program progressed over the 12-week period as the women became more technically skilled and fitness levels improved.

\section{Statistical Analysis}

Baseline data are presented as mean and standard deviation for continuous data and number and percentage for categorical data. Change of data were calculated as post values minus pre values. A paired t-test was used to assess change in body composition and cardiometabolic risk factors after a 12-week aerobic exercise program. The following variables were natural log transformed prior to analysis as assessed by P-P plots; SAAT, body fat, apolipoprotein a, apolipoprotein b, cholesterol, triglycerides, high density lipoprotein cholesterol, low density lipoprotein cholesterol, non HDL cholesterol, aluminotransferase, C reactive protein, glucose, insulin, HOMA-IR. To answer our primary objective, Pearson 
bivariate correlations were used to assess the association between change in body composition and change in cardio-metabolic risk factors with change in the independent variables of interest; VAT, SAAT, WC, body fat and BMI. To answer our secondary objective, separate linear regression models were created for those risk factors that were correlated with VAT as the dependent variables and VAT, SAAT, WC, body fat and BMI as the independent variables. Age and change in $\mathrm{VO}_{2}$ peak were included as covariates to assess whether associations were independent of change in aerobic fitness. Figures 1 and 2 were created in order to model the inter individual response as done by other exercise studies (King et al., 2007 and 2008). To answer our tertiary objective, models with VAT and SAAT were additionally adjusted for change in WC in order to determine whether the associations of VAT and SAAT with cardio-metabolic risk factors were independent of WC. As well, the model with body fat was additionally adjusted for change in BMI, to assess whether the associations with cardio-metabolic risk factors were independent of BMI. Statistical significance was set at $<0.05$. Analyses were conducted using SPSS Version 23.0.

\section{RESULTS}

The following results are a sub analysis of the data from the previously published randomized controlled trial from which this population was contrived. We have previously found in within-group pre-post comparisons, that there was a significant reduction in VAT $(\mathrm{p}=0.040)$ and $\mathrm{WC}(\mathrm{p}=0.037)$ after a 12 -week standard exercise program, as well as in SAAT $(\mathrm{p}<0.001)$, TAAT $(\mathrm{P}<0.001)$, BMI $(\mathrm{p}=0.027), \mathrm{WC}(\mathrm{p}<0.001)$, body fat $(\mathrm{p}=0.036)$ and glucose $(p=0.039)$ after a 12-week Bhangra dance program (Lesser et al., 2016).

Forty-nine women participated in the exercise programs and had mean (SD) age of $57 \pm 6$ years. Women were predominantly from India (87.8\%) and spoke Punjabi (77.6\%). Two 
women had impaired fasting glucose as determined by International Diabetes Federation guidelines of greater than $7.0 \mathrm{mmol} / \mathrm{L}$. Adherence to the exercise interventions was based on attendance at the 36 prescribed exercise classes and averaged 72\%. Table 1 shows the pre and post data for abdominal adiposity, body composition and aerobic fitness.

There was a significant reduction after exercise training in VAT, TAAT, SAAT, waist circumference, body fat percent, and glucose and a significant improvement in $\mathrm{VO}_{2}$ peak $(\mathrm{p}<0.05)$. Figure 1 depicts the inter-individual change in VAT, SAAT and WC over the course of the 12-week intervention in ascending order of change in VAT. There were more individuals who had the same direction of change as VAT when assessing SAAT compared to WC.

Figure 2 depicts the inter-individual change in body fat over the course of the 12 -week intervention in ascending order of body fat matched with BMI. There appeared to be a similar pattern of change in body fat with change in BMI.

Correlations between change in body composition and cardio-metabolic risk factors with change in body composition variables of interest; VAT, SAAT, WC, BMI and body fat are presented in Table 2 .

There were significant positive associations between change in VAT and change in WC with change in glucose $(r=0.499, p<0.001$ and $r=0.335, p=0.020)$, insulin $(r=0.452, p<0.001$ and $\mathrm{r}=0.480, \mathrm{p}=0.001)$ and HOMA-IR $(\mathrm{r}=0.604, \mathrm{p}<0.001$ and $\mathrm{r}=0.385, \mathrm{p}=0.007)$ and change in BMI with change in insulin $(r=0.341, p=0.018)$ and HOMA-IR $(r=0.436, p=0.002)(p<0.05)$ (Table 2). In addition, change in VAT was significantly associated with change in BMI $(\mathrm{r}=0.551, \mathrm{p}<0.001)$ and body fat $(\mathrm{r}=0.306, \mathrm{p}=0.032)$ but not change in WC $(\mathrm{r}=0.189$, 
$\mathrm{p}=0.193$ ) or SAAT $(\mathrm{r}=0.217, \mathrm{p}=0.139)$ while there was a significant association between change in BMI and change in WC $(r=0.284, p=0.048)$.

Separate multiple linear regression analyses with change in VAT, SAAT, WC, BMI and body fat as the independent variables and glucose, insulin and HOMA-IR as the dependent variables adjusted for age and change in $\mathrm{VO}_{2}$ peak were conducted. There was a significant association with change in VAT and change in glucose $(\beta=0.451, p=0.004)$, insulin $(\beta=$ 0.548, $\mathrm{p}<0.001)$ and HOMA-IR $(\beta=0.639, \mathrm{p}<0.001)$. These associations remained after adjustment for change in WC or change in SAAT. There was a minimally statistically significant association between change in WC and change in HOMA-IR $(\beta=0.347, p=0.036)$ and glucose $(\beta=0.452, p=0.005)$ but no statistically significant change in insulin $(\beta=0.279$, $\mathrm{p}=0.094)$ (Table 3).

There were no significant associations between change in body fat and change in glucose $(\beta$ $=0.227 \mathrm{p}=0.175)$, insulin $(\beta=0.077 \mathrm{p}=0.651)$ or HOMA-IR $(\beta=0.182 \mathrm{p}=0.279)$ after adjustment for age or change in $\mathrm{VO}_{2}$ peak. Further adjustment for change in BMI did not alter these results ( $\mathrm{p}>0.05)$. 


\section{DISCUSSION}

A 12-week exercise program in previously inactive post-menopausal women reduced VAT, SAAT, TAAT, WC, body fat and fasting blood glucose and improved aerobic fitness. Bivariate correlations showed significant associations between both change in VAT and change in WC with change in glucose, insulin and HOMA-IR. As well, change in BMI correlated with change in insulin and HOMA-IR. The relationship between change in VAT and change in glucose, insulin and HOMA-IR remained significant after adjustment for age and aerobic fitness, and also after further adjustment for change in WC or change in SAAT. Change in WC was only significantly associated with change in HOMA-IR after adjustment for age and change in aerobic fitness.

Previous research has shown that WC is the best indicator of VAT and related CVD risk (Pouliot et al. 1994) but whether this remains true after an exercise intervention is not well studied. In a weight loss study, Pare et al. (2001) found change in VAT was associated with change in WC; however, this study did not assess change in cardio-metabolic risk. In the current study, the association between changes in VAT and changes in glucose, insulin and HOMA-IR remained statistically significant even after adjusting for change in WC; and change in VAT was not associated with change in WC. A similar study in a Japanese population found change in VAT to be significantly associated with the change in cardiometabolic risk factors, while no such association was observed for WC; the authors suggested that change in WC may not be a good indicator of weight loss and improvement in metabolic risk factors (Yamakage et al. 2014). The results of our study also indicate that change in VAT is a better indicator of an improvement in markers of glucose regulation than change in WC in this population of post-menopausal South Asian women. We found that change in WC was only associated with change in HOMA-IR but not with changes in glucose 
or insulin. This may be due to a non clinically significant reduction in glucose and insulin with aerobic exercise training.

Our results are similar to previous exercise studies researching the relationships between changes in adiposity measures and insulin sensitivity in European populations that have shown that reductions in VAT are associated with improvements in insulin sensitivity (O’Leary et al., 2006; Nicklas et al., 2009; Gan et al., 2003; Cnop et al., 2002). We extend the existing body of knowledge by reporting these relationships in post-menopausal South Asian women, a population at high risk for CVD and diabetes who possess a unique obesity phenotype. Similar to the results of our study, Cnop et al. (2002), found a significant association between change in VAT and change in insulin resistance, and this association remained independent of BMI. Furthermore, VAT was reported as an independent risk factor for insulin resistance even after taking into account SAAT (Ross et al. 2002). We also found the change in VAT to be significantly associated with change in markers of glucose regulation, independent of change in SAAT. Visceral adipose tissue has previously been shown to explain the elevated cardio-metabolic risk in the South Asian population compared to Europeans (Lear et al., 2012). However, the study was cross-sectional and did not determine whether altering VAT through aerobic exercise could improve cardiometabolic risk in the South Asian population. The association between VAT reduction and improvements in markers of glucose regulation provides support for aerobic exercise in the South Asian population to alter the high prevalence of T2D and CVD (Gholap et al., 2011), as those who do reduce their VAT deposition through aerobic exercise may see improvements in HOMA-IR.

Limitations of this study include the crude measurement of insulin sensitivity through HOMA-IR. While fasting insulin measures have been shown to be a useful surrogate for insulin sensitivity (Lorenzo et al. 2010), it would have been beneficial to have a 
measurement of both peripheral and hepatic insulin sensitivity using the hyperinsulinemiceuglycemic clamp method in conjunction with an isotopic tracer (Dunn et al. 2012). In addition, the study sample was limited to a homogenous population of abdominally obese, post-menopausal South Asian women and therefore results cannot be generalized to South Asian men or pre menopausal women. Despite the limitations, this study used the gold standard of CT imaging for the assessment of VAT and provides novel information regarding the role of exercise on obesity and cardio-metabolic risk in a population of postmenopausal South Asian women.

In conclusion, we found statistically significant associations between change in VAT with changes in fasting insulin, glucose and HOMA-IR in post-menopausal South Asian women after a 12- week aerobic exercise training program. The association between change in VAT and these cardio-metabolic risk factors was independent of change in WC and SAAT and therefore a reduction in VAT may be an important indicator of improvements in glucose regulation despite not seeing a reduction in WC. South Asian women should be encouraged to engage in aerobic activity to reduce their risk of type 2 diabetes and CVD, and physicians should be aware of improvements in glucose regulation that may be seen with exercise training that are not observed through reductions in WC.

\section{ACKNOWLEDGEMENTS}

IAL is supported by the SANSAR Young Investigator Award. SAL holds the Pfizer Heart and Stroke Foundation Chair in Cardiovascular Prevention Research at St. Paul's Hospital. This research was funded by a grant from the Canadian Institutes of Health Research.

\section{CONFLICT OF INTEREST STATEMENT}

The authors declare that there are no conflicts of interest. 


\section{REFERENCES}

Alberti, K. G., Zimmet, P., Shaw, J., \& IDF Epidemiology Task Force Consensus Group. 2005. The metabolic syndrome--a new worldwide definition. Lancet, 366 (9491), 1059-1062.

Arner, P., Kriegholm, E., Engfeldt, P., \& Bolinder, J. 1990. Adrenergic regulation of lipolysis in situ at rest and during exercise. The Journal of Clinical Investigation, 85(3), 893-898.

Arsenault, B. J., Cote, M., Cartier, A., Lemieux, I., Despres, J. P., Ross, R., et al. 2009. Effect of exercise training on cardiometabolic risk markers among sedentary, but metabolically healthy overweight or obese post-menopausal women with elevated blood pressure. Atherosclerosis, 207(2), 530-533.

Borel, A. L., Nazare, J. A., Smith, J., Almeras, N., Tremblay, A., Bergeron, J., et al. 2012. Improvement in insulin sensitivity following a 1-year lifestyle intervention program in viscerally obese men: Contribution of abdominal adiposity. Metabolism: Clinical and Experimental, 61(2), 262-272.

Bouchonville, M., Armamento-Villareal, R., Shah, K., Napoli, N., Sinacore, D. R., Qualls, C., et al. 2014. Weight loss, exercise or both and cardiometabolic risk factors in obese older adults: Results of a randomized controlled trial. International Journal of Obesity (2005), 38(3), 423-431.

Bruce, R. A., Kusumi, F., \& Hosmer, D. 1973. Maximal oxygen intake and nomographic assessment of functional aerobic impairment in cardiovascular disease. American Heart Journal, 85(4), 546-562. 
Cnop, M., Landchild, M. J., Vidal, J., Havel, P. J., Knowles, N. G., Carr, D. R., et al. 2002. The concurrent accumulation of intra-abdominal and subcutaneous fat explains the association between insulin resistance and plasma leptin concentrations : Distinct metabolic effects of two fat compartments. Diabetes, 51(4), 1005-1015.

Despres, J. P. 2014. Waist circumference as a vital sign in cardiology 20 years after its initial publication in the american journal of cardiology. The American Journal of Cardiology, 114(2), 320-323.

Dunn, J. P., Abumrad, N. N., Breitman, I., Marks-Shulman, P. A., Flynn, C. R., Jabbour, K., et al. 2012. Hepatic and peripheral insulin sensitivity and diabetes remission at 1 month after roux-en-Y gastric bypass surgery in patients randomized to omentectomy. Diabetes Care, 35(1), 137-142.

Fabbrini, E., Tamboli, R. A., Magkos, F., Marks-Shulman, P. A., Eckhauser, A. W., Richards, W. O., et al. 2010. Surgical removal of omental fat does not improve insulin sensitivity and cardiovascular risk factors in obese adults. Gastroenterology, 139(2), 448-455.

Fried, S.K., Leibel, R.L., Edens, N.K. \& Kral, J.G. 1993. Lipolysis in intraabdominal adipose tissues of obese women and men. Obesity research, 1(6), 443-448.

Friedewald, W. T., Levy, R. I., \& Fredrickson, D. S. 1972. Estimation of the concentration of low-density lipoprotein cholesterol in plasma, without use of the preparative ultracentrifuge. Clinical Chemistry, 18(6), 499-502.

Gan, S. K., Kriketos, A. D., Ellis, B. A., Thompson, C. H., Kraegen, E. W., \& Chisholm, D. J. 2003. Changes in aerobic capacity and visceral fat but not myocyte lipid levels predict 
increased insulin action after exercise in overweight and obese men. Diabetes Care, 26(6), 1706-1713.

Garduno-Diaz, S. D., \& Khokhar, S. 2011. Prevalence, risk factors and complications associated with type 2 diabetes in migrant south asians. Diabetes Metab. Res. Rev, $28(1): 6-24$

Gholap, N., Davies, M., Patel, K., Sattar, N., \& Khunti, K. 2011. Type 2 diabetes and cardiovascular disease in south asians. Primary Care Diabetes, 5(1), 45-56.

Janiszewski PM, and Ross R. (2009). The utility of physical activity in the management of global cardiometabolic risk. Obesity 3:S3-S14.

Janssen, I., Powell, L. H., Jasielec, M. S., \& Kazlauskaite, R. 2015. Covariation of change in bioavailable testosterone and adiposity in midlife women. Obesity (Silver Spring, Md.), 23(2), 488-494.

King, N.A., Caudwell P., Hopkins, M., Byrne, N.M., Colley, R., Hills, A.P., Stubbs, H.R. and Blundell, J.E. 2007. Metabolic and behavioral compensatory responses to exercise interventions: barriers to weight loss. Obesity. 15(6), 1373-83.

King, N.A., Hopkins, M., Caudwell, P., Stubbs, J.R. and Blundell, J.E. 2008. Individual variability following 12 weeks of supervised exercise: identification and characterization or compensation for exercise-induced weight loss. Int. J. Obes, 32, 17784. 
Lear, S. A., Chockalingam, A., Kohli, S., Richardson, C. G., \& Humphries, K. H. 2012. Elevation in cardiovascular disease risk in south asians is mediated by differences in visceral adipose tissue. Obesity (Silver Spring, Md.), 20(6): 1293-300.

Lear, S. A., Humphries, K. H., Kohli, S., Chockalingam, A., Frohlich, J. J., \& Birmingham, C. L. 2007. Visceral adipose tissue accumulation differs according to ethnic background: Results of the multicultural community health assessment trial (M-CHAT). Am. J. Clin. Nutr, 86(2), 353-359.

Lear, S. A., Kohli, S., Bondy, G. P., Tchernof, A., \& Sniderman, A. D. (2009). Ethnic variation in fat and lean body mass and the association with insulin resistance. The Journal of Clinical Endocrinology and Metabolism, 94(12), 4696-4702.

Lemieux, I., Pascot, A., Couillard, C., Lamarche, B., Tchernof, A., Almeras, N., et al. 2000. Hypertriglyceridemic waist: A marker of the atherogenic metabolic triad (hyperinsulinemia; hyperapolipoprotein B; small, dense LDL) in men? Circulation, 102(2), 179-184.

Lesser, I.A., Singer, J., Hoogbruin A., Mackey, D.C., Katzmarzyk., P.T., Sohal, P., Leipsic, J., \& Lear, S.A. 2016. Effectiveness of Exercise on Visceral Adipose Tissue in Older South Asian Women. Am. J. Sports Med, Published ahead of print.

Lorenzo, C., Haffner, S. M., Stancakova, A., \& Laakso, M. 2010. Relation of direct and surrogate measures of insulin resistance to cardiovascular risk factors in nondiabetic finnish offspring of type 2 diabetic individuals. J. Clin. Endocrinol. Metab, 95(11), 50825090. 
Nicklas, B. J., Wang, X., You, T., Lyles, M. F., Demons, J., Easter, L., et al. 2009. Effect of exercise intensity on abdominal fat loss during calorie restriction in overweight and obese postmenopausal women: A randomized, controlled trial. Am. J. Clin. Nutr, 89(4), 10431052.

O'Leary, V. B., Marchetti, C. M., Krishnan, R. K., Stetzer, B. P., Gonzalez, F., \& Kirwan, J. P. 2006. Exercise-induced reversal of insulin resistance in obese elderly is associated with reduced visceral fat. J. Appl. Physiol. (Bethesda, Md.: 1985), 100(5), 1584-1589.

Pare, A., Dumont, M., Lemieux, I., Brochu, M., Almeras, N., Lemieux, S., et al. 2001. Is the relationship between adipose tissue and waist girth altered by weight loss in obese men? Obes. Research, 9(9), 526-534.

Pouliot, M. C., Despres, J. P., Lemieux, S., Moorjani, S., Bouchard, C., Tremblay, A., et al. 1994. Waist circumference and abdominal sagittal diameter: Best simple anthropometric indexes of abdominal visceral adipose tissue accumulation and related cardiovascular risk in men and women. Am. J. Cardiol, 73(7), 460-468.

Preis, S. R., Massaro, J. M., Robins, S. J., Hoffmann, U., Vasan, R. S., Irlbeck, T., et al. 2010. Abdominal subcutaneous and visceral adipose tissue and insulin resistance in the framingham heart study. Obesity (Silver Spring, Md.), 18(11), 2191-2198.

Rosano, G. M., Vitale, C., Marazzi, G., \& Volterrani, M. 2007. Menopause and cardiovascular disease: The evidence. Climacteric, 10 Suppl 1, 19-24.

Ross, R., Aru, J., Freeman, J., Hudson, R., \& Janssen, I. 2002. Abdominal adiposity and insulin resistance in obese men. Am. J. Physiol. .Endocrinol. Metabol, 282(3), E657-63. 
Yamakage, H., Ito, R., Tochiya, M., Muranaka, K., Tanaka, M., Matsuo, Y., et al. 2014. The utility of dual bioelectrical impedance analysis in detecting intra-abdominal fat area in obese patients during weight reduction therapy in comparison with waist circumference and abdominal CT. Endocrine Journal, 61(8), 807-819. 
Table 1. Paired t-tests assessing pre-post change in abdominal fat, body composition, cardio-metabolic risk factors and aerobic fitness $n=49$

\begin{tabular}{|l|c|c|c|}
\hline & Pre & Post & P-value \\
\hline Visceral Adipose Tissue $\left(\mathrm{cm}^{3}\right)$ & & & \\
\hline Total Abdominal Adipose Tissue $\left(\mathrm{cm}^{3}\right)$ & $2665 \pm 1039$ & $2591 \pm 1039$ & 0.017 \\
\hline Subcutaneous Abdominal Adipose Tissue $\left(\mathrm{cm}^{3}\right)$ & $6093 \pm 2577$ & $8860 \pm 2684$ & 0.003 \\
\hline Body Mass Index (kg/m $\left.{ }^{2}\right)$ & $30.0 \pm 4.1$ & $29.8 \pm 4.2$ & 0.199 \\
\hline Waist Circumference (cm) & $94.8 \pm 9.2$ & $92.4 \pm 9.2$ & 0.001 \\
\hline Body Fat (\%) & $43.8 \pm 4.6$ & $43.0 \pm 4.6$ & 0.021 \\
\hline Apolipoprotein A (g/L) & $1.60 \pm 0.24$ & $1.57 \pm 0.28$ & 0.423 \\
\hline Apolipoprotein B (g/L) & $0.98 \pm 0.30$ & $0.92 \pm 0.33$ & 0.121 \\
\hline Total Cholesterol (mmol/L) & $5.17 \pm 1.18$ & $5.03 \pm 1.01$ & 0.197 \\
\hline Triglycerides (mmol/L) & $1.43 \pm 0.91$ & $1.37 \pm 0.66$ & 0.446 \\
\hline High Density Lipoprotein Cholesterol (mmol/L) & $1.56 \pm 0.40$ & $1.54 \pm 0.38$ & 0.340 \\
\hline Low Density Lipoprotein Cholesterol (mmol/L) & $2.90 \pm 0.86$ & $2.87 \pm 0.83$ & 0.691 \\
\hline Non High Density Lipoprotein Cholesterol & $3.41 \pm 1.09$ & $3.45 \pm 0.99$ & 0.742 \\
\hline Aluminotransferase (U/L) & $24.5 \pm 11.3$ & $23.1 \pm 8.2$ & 0.248 \\
\hline C- Reactive Protein (mg/L) & $2.4 \pm 1.7$ & $2.7 \pm 1.9$ & 0.055 \\
\hline Glucose (mmol/L) & $5.67 \pm 0.77$ & $5.43 \pm 0.49$ & 0.004 \\
\hline Insulin (pmol/L) & $93.7 \pm 41.6$ & $91.6 \pm 53.0$ & 0.741 \\
\hline Homeostatic Model Assessment of Insulin & $3.5 \pm 1.8$ & $3.3 \pm 2.1$ & 0.403 \\
\hline Peak Oxygen Uptake (mL/kg/min) & $22.8 \pm 3.7$ & $24.7 \pm 4.5$ & $<0.001$ \\
\hline Time to Exhaustion (minutes) & $5.1 \pm 1.2$ & $5.5 \pm 1.8$ & 0.115 \\
\hline
\end{tabular}

Significance set at $\mathrm{p}<0.05$

Change data were calculated as post values minus pre values. Data are presented as means and standard deviations.

All blood measurements were taken after a 12 hour overnight fast. 
Table 2. Pearson bivariate correlations between change in body composition variables of interest and change in cardio-metabolic risk factors

\begin{tabular}{|c|c|c|c|c|c|}
\hline & $\begin{array}{l}\Delta \text { Visceral Adipose } \\
\text { Tissue }\end{array}$ & $\begin{array}{l}\Delta \text { Subcutaneous } \\
\text { Abdominal Adipose } \\
\text { Tissue } *\end{array}$ & $\begin{array}{l}\Delta \text { Waist } \\
\text { Circumference }\end{array}$ & $\Delta$ Body Fat & $\Delta$ Body Mass Index \\
\hline $\begin{array}{l}\Delta \text { Total Abdominal Adipose } \\
\text { Tissue }\end{array}$ & $0.573^{* * *}$ & $0.689 * * *$ & $0.371^{* *}$ & 0.197 & $0.579 * * *$ \\
\hline$\Delta$ Visceral Adipose Tissue & - & 0.217 & 0.189 & $0.306^{*}$ & $0.551 * * *$ \\
\hline $\begin{array}{l}\Delta \text { Subcutaneous Abdominal } \\
\text { Adipose Tissue }\end{array}$ & 0.217 & - & 0.166 & 0.169 & $0.431^{* *}$ \\
\hline$\Delta$ Body Mass Index & $0.551 * * *$ & 0.431 ** & $0.284 *$ & $0.487 * * *$ & - \\
\hline$\Delta$ Waist Circumference & 0.189 & 0.166 & - & -0.164 & $0.284^{*}$ \\
\hline$\Delta$ Body Fat & $0.306^{*}$ & +2 & -0.153 & - & $0.452 * *$ \\
\hline$\Delta$ Body Fat Percent & 0.125 & 0.054 & $-0.310^{*}$ & $0.924 * * *$ & 0.192 \\
\hline$\Delta$ Apolipoprotein A & 0.069 & 0.078 & 0.134 & -0.054 & 0.143 \\
\hline$\Delta$ Apolipoprotein B & -0.226 & -0.058 & 0.129 & $-0.315^{*}$ & -0.154 \\
\hline$\Delta$ Total Cholesterol & -0.151 & -0.075 & 0.005 & -0.008 & -0.097 \\
\hline$\Delta$ Triglycerides & -0.094 & -0.087 & 0.109 & 0.045 & -0.019 \\
\hline $\begin{array}{l}\Delta \text { High Density Lipoprotein } \\
\text { Cholesterol }\end{array}$ & 0.061 & -0.075 & 0.025 & 0.152 & 0.132 \\
\hline $\begin{array}{l}\Delta \text { Low Density Lipoprotein } \\
\text { Cholesterol }\end{array}$ & -0.112 & 0.027 & 0.210 & -0.164 & -0.018 \\
\hline $\begin{array}{l}\Delta \text { Non High Density Lipoprotein } \\
\text { Cholesterol }\end{array}$ & 0.136 & -0.068 & -0.015 & -0.027 & -0.145 \\
\hline
\end{tabular}




\section{Applied Physiology, Nutrition, and Metabolism}

\begin{tabular}{|l|l|l|l|l|l|}
\hline$\Delta$ Aluminotransferase & 0.180 & 0.043 & 0.268 & 0.067 & 0.232 \\
\hline$\Delta$ C Reactive Protein & 0.084 & 0.049 & 0.152 & 0.173 & 0.273 \\
\hline$\Delta$ Glucose & $0.499^{* * *}$ & -0.030 & $0.335^{*}$ & 0.208 & 0.271 \\
\hline$\Delta$ Insulin & $0.452^{* * *}$ & 0.162 & $0.480^{* *}$ & 0.054 & $0.341^{*}$ \\
\hline $\begin{array}{l}\Delta \text { Homeostatic Model } \\
\text { Assessment of Insulin Resistance }\end{array}$ & $0.604^{* * *}$ & 0.140 & $0.385^{* *}$ & 0.161 & $0.436^{* *}$ \\
\hline
\end{tabular}

${ }^{*} \mathrm{p}<0.05,{ }^{* *} \mathrm{p}<0.01,{ }^{* * *} \mathrm{p}<0.001$

Change data presented as post value minus pre value 
Table 3. Multiple regression analyses for body composition variables of interest with glucose, insulin and homeostatic model assessment of insulin resistance

\begin{tabular}{|c|c|c|c|}
\hline & $\Delta$ Visceral Adipose Tissue & $\begin{array}{l}\Delta \text { Subcutaneous Abdominal } \\
\text { Adipose Tissue }\end{array}$ & $\Delta$ Waist Circumference \\
\hline \multicolumn{4}{|l|}{ Glucose } \\
\hline Model 1a & $0.451^{* *}$ & 0.121 & $0.452 * *$ \\
\hline Model 1b & $0.421^{* *}$ & -0.138 & - \\
\hline Model 1c & $0.489 * *$ & - & - \\
\hline \multicolumn{4}{|l|}{ Insulin } \\
\hline Model 2a & $0.548 * * *$ & 0.189 & 0.279 \\
\hline Model 2b & $0.478^{* *}$ & 0.173 & - \\
\hline Model 2c & $0.539 * *$ & - & - \\
\hline \multicolumn{4}{|l|}{ HOMA-IR } \\
\hline Model 3a & $0.639 * * *$ & 0.185 & $0.347^{*}$ \\
\hline Model 3b & $0.603 * * *$ & -0.011 & - \\
\hline Model 3c & $0.642 * * *$ & - & - \\
\hline
\end{tabular}

*represents $\mathrm{p}<0.05,{ }^{* *}$ represents $\mathrm{p}<0.01,{ }^{* * *}$ represents $\mathrm{p}<0.001$

Change data presented as post value minus pre value

Independent variables of interest are change in glucose, insulin and HOMA-IR. Dependent variables of interest are change in visceral adipose tissue (VAT), subcutaneous abdominal adipose tissue (SAAT) and waist circumference.

Model a: Adjusted for age and change in $\mathrm{VO}_{2}$ peak, Model b: Models with VAT and SAAT additionally adjusted for Waist Circumference for VAT and SAAT, Model c: Models with VAT and WC additionally adjusted for SAAT Values displayed are regression coefficients. 
Figure 1. (a) Individual changes in visceral adipose tissue (VAT) volume are represented by decreasing order, (b) Individual changes in subcutaneous abdominal adipose tissue (SAAT) in the same order as histogram A, (c) Individual changes in waist circumference (WC) in the same order as histogram A

Figure 2. (a) Individual changes in body fat are represented by decreasing order, (b) Individual changes in body mass index (BMI) in the same order as histogram A 

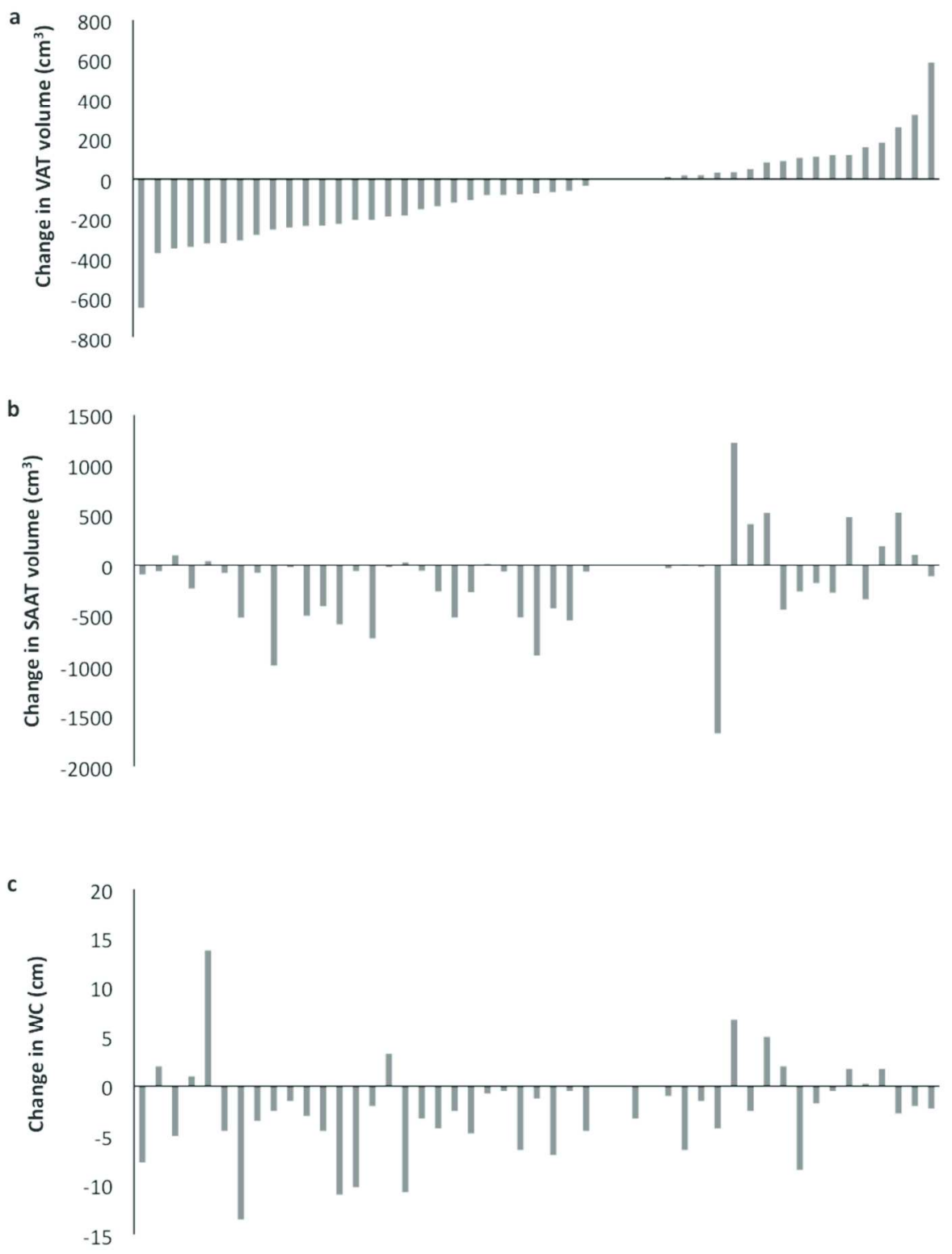

$152 \times 196 \mathrm{~mm}(300 \times 300$ DPI $)$ 

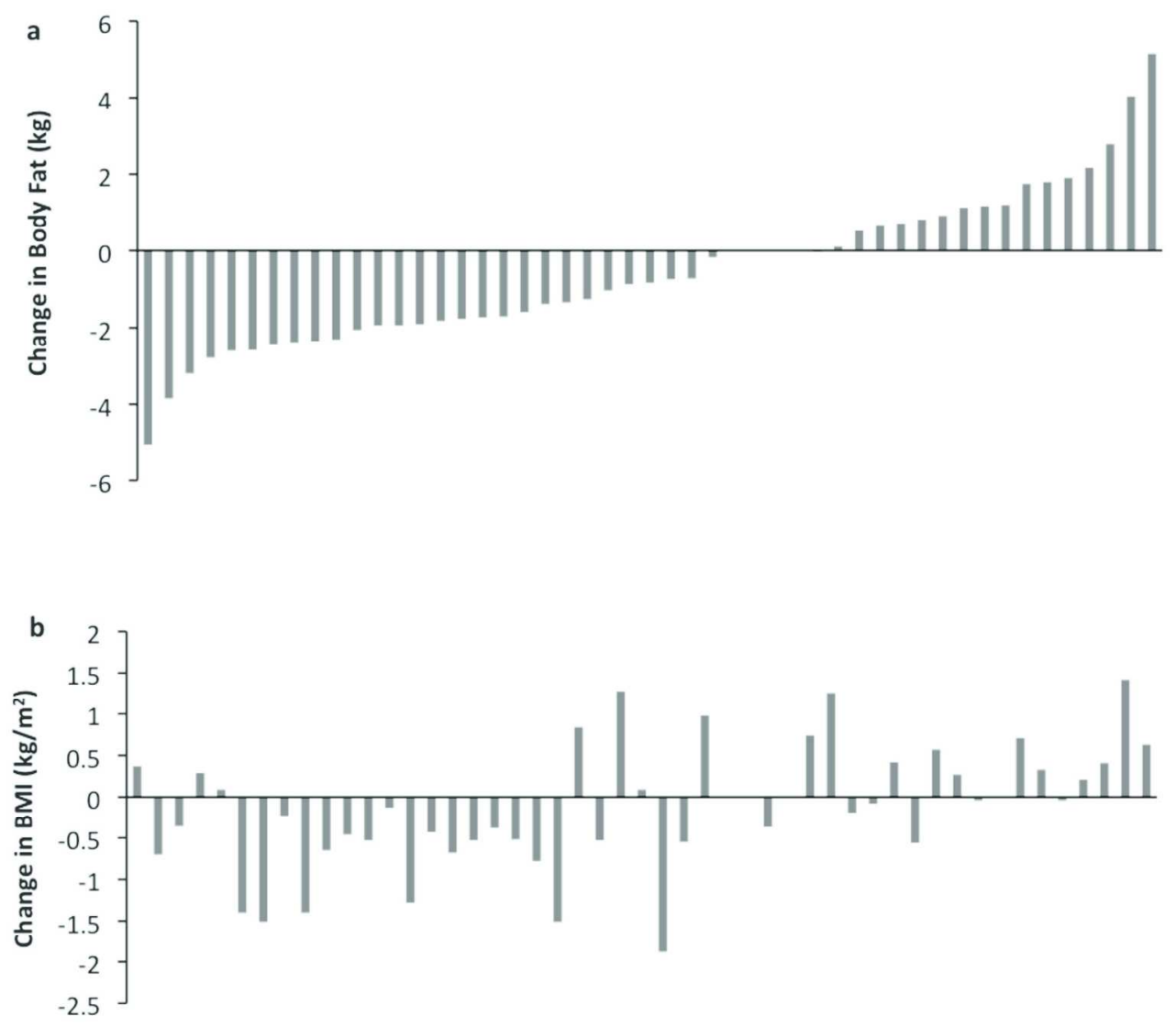

$152 \times 135 \mathrm{~mm}(300 \times 300$ DPI $)$ 